\title{
Mitteilungen aus der Bundesdirektorenkonferenz (BDK)
}

Verantwortlich für diese Rubrik: Gerhard Längle, Tübingen/Bad Schussenried; Thomas Pollmächer, Ingolstadt

\section{Der Arbeitskreis „Psychotherapie“ $\nabla$}

Psychotherapie ist nicht erst seit der Aufnahme der Psychotherapie in die Bezeichnung des Facharztes für Psychiatrie und Psychotherapie 1992 eine Kernkompetenz in der psychiatrischen Behandlung. Bereits seit vielen Jahrzehnten widmen sich nicht nur spezifische Stationen und Abteilungen der psychiatrischen Fachkrankenhäuser diesem Thema, es ist uns in den letzten Jahren auch gelungen, in den Akutbereichen psychotherapeutische Konzepte zu entwickeln, die motivationale Therapieinterventionen in den Vordergrund rücken. Hierbei ist psychotherapeutische Expertise nicht nur für die bessere Behandlung der Patienten von großer und wachsender Bedeutung, auch für die Weiterbildung der Mitarbeiter und in der Außenwirkung der Kliniken spielen diese Aspekte eine wichtige Rolle.

Trotz der in Deutschland existierenden großen Anzahl von Kliniken für Psychosomatische Medizin und Psychotherapie erfolgen noch immer weit überwiegend Behandlungen psychischer Störungen im Bereich psychiatrischer Fachkrankenhäuser. Neben den auch in psychosomatischen Fachkliniken behandelten Patienten mit affektiven Störungen, Angststörungen, somatoformen Störungen und EssStörungen werden dabei in den psychiatrischen Kliniken auch in großem Umfang Behandlung von schweren Persönlichkeitsstörungen, psychotischen Störungen und Suchterkrankungen durchgeführt, außerdem existiert eine breite gerontopsychiatrische Expertise. Betrachtet man die Patienten der Psychotherapiestationen und -abteilungen der psychiatrischen Fachkrankenhäuser, so zeigt sich hier ein vergleichbares Diagnosespektrum wie in den Kliniken für Psychosomatische Medizin und Psychotherapie, allerdings oft ein höheres Maß an Chronifizierung und komorbiden Störungen. Darüber hinaus sind auch in den Bereichen für Krisenbehandlungen, Suchtbehandlungen und in der Alterspsychiatrie psychothe- rapeutische Behandlungskonzepte entwickelt worden. Hieraus leitet sich zum einen die Forderung ab, weiterhin auch in psychiatrischen Fachkliniken psychotherapeutische Behandlungskonzepte für affektive Störungen, Angststörungen, Essstörungen und somatoformen Störungen vorzuhalten und auszubauen. Zum anderen besteht aber auch ein Bedarf zur Weiterentwicklung von psychotherapeutischen Konzepten für Patienten mit Psychosen, gerontopsychiatrische Patienten und Suchterkrankte sowohl in akuten, oft von Ambivalenz geprägten Aufnahmesituationen, als auch im weiteren Verlauf in elektiven Behandlungsprogrammen. Im Vorfeld der Herbsttagung der BDK 2013 in Wasserburg war eine Umfrage zur Psychotherapie unter den Mitgliedern erfolgt: Neben Fragen zur strukturellen Organisation der Psychotherapieangebote wurden auch solche nach deren inhaltlichen und therapeutischen Ausrichtung, den damit befassten Berufsgruppen und der Bedeutung psychotherapeutischer Weiterbildung in den Weiterbildungsangeboten der Häuser gestellt. Schließlich wurden die Mitglieder der Bundesdirektorenkonferenz bzgl. der zukünftigen inhaltlichen Ausrichtung eines Arbeitskreises Psychotherapie in der BDK befragt. Hier wurden durch die Mitglieder vor allen Dingen eine verbesserte Außendarstellung der bereits bestehenden psychotherapeutischen Kompetenzen der Kliniken gefordert. Es wurde die Weiterentwicklung von Therapiekonzepten insbesondere für Suchtpatienten, gerontopsychiatrische Patienten und Psychosepatienten für nötig erachtet, die derzeit im Bereich der Richtlinienpsychotherapie und in psychosomatischen Kliniken nur in geringem Umfang behandelt werden. Außerdem wurde vorgeschlagen, Therapiekonzepte zu erarbeiten und zu evaluieren, die versuchen, psychotherapeutische Behandlungen im stationären, teilstationären und ambulanten Setting zu verknüpfen und den Patienten wohnortnah zur Verfügung zu stellen. Schließlich ist der Arbeitskreis aufgefordert worden, sich inhaltlich um die Vereinheitlichung der ärztlich-psychotherapeutischen Weiterbildung und um den Aufbau psychotherapeutischer Kompetenzen, insbesondere im Bereich des Fachpflegepersonals zu kümmern.

Nach einer ersten konstituierenden Sitzung des wiedergegründeten Arbeitskreises bei der Herbsttagung 2013 in Wasserburg erfolgte dann im Frühjahr 2014 eine erste, gut besuchte Versammlung mit Arbeitskreismitglieder aus ganz Deutschland. Tagungsort war die Vitos Klinik in Kassel-Wilhelmshöhe, neben einer großen Anzahl leitender Ärzte haben auch leitende Psychologen aus unterschiedlichen Klinken daran teilgenommen. Neben organisatorischen Fragen wie der Bestimmung eines Sprechergremiums widmete sich der Arbeitskreis unter anderem Fragen zur Rolle der Psychotherapie-Weiterbildung in der neuen Facharztausbildung. Fachvorträge am Nachmittag beschäftigten sich mit Psychotherapieangeboten für spezifische Patientengruppen, die derzeit in der Richtlinienpsychotherapie eine eher nachgeordnete Rolle spielen: So stellte Prof. Wiedemann aus Fulda in einem Überblicksvortrag psychotherapeutische Konzepte zur Behandlung von Patienten mit Psychosen dar, während Dr. Reker aus Bielefeld in seinem Vortrag neue Konzepte zur Psychotherapie von alkoholabhängigen Patienten vorstellte.

Mittlerweile hat der Arbeitskreis auch einen Internetauftritt im Rahmen der BDK-Homepage erstellt. Hier kann die breite psychotherapeutische Expertise der einzelnen Mitglieder zum einen an dort dargestellten wissenschaftlichen Publikationen der Mitglieder abgelesen werden, zum anderen existiert hier auch die Möglichkeit, Referenten zu spezifischen psychotherapeutischen Themen $z u$ finden.

Andreas Schuld, Ingolstadt Arndt Heinemann, Magdeburg Martin Reker, Bielefeld 